\title{
Influência de tecnologias para avaliação/visualização vascular no cateterismo intravenoso periférico: Revisão integrativa
}

\author{
Influence of vascular assessment/visualization in peripheral intravenous catheterization technologies:
} An integrative review

Influencia de tecnologías para la evaluación/visualización vascular en el cateterismo intravenoso periférico: Revisión integrativa

\section{Luciano Marques dos Santos ${ }^{1,2}$ (c) Sarah Almeida Santos ${ }^{1}$ (1) Bianka Souza Martins Silva² (1) Rosana Castelo Branco de Santana ${ }^{3}$ (C) Ariane Ferreira Machado Avelar ${ }^{2}$ (B)}

1. Universidade Estadual de Feira de Santana. Feira de Santana, BA, Brasil.

2. Universidade Federal de São Paulo. São Paulo, SP, Brasil.

3. Universidade Federal da Bahia. Salvador, BA, Brasil.
Autor correspondente:

Sarah Almeida Santos.

E-mail: sarahalmeida21@hotmail.com.

Recebido em 12/12/2019.

Aprovado em 28/02/2020.

DOI: 10.1590/2177-9465-EAN-2019-0355

\section{RESUMO}

Objetivo: verificar a influência da ultrassonografia vascular, emissão de luz infravermelha e iluminação transdérmica no sucesso da cateterização intravenosa periférica; número de tentativas, tempo para a realização do procedimento, permanência do cateter in situ e ocorrência de complicações em crianças, quando comparadas ao método tradicional. Método: trata-se de uma revisão integrativa da literatura realizada no período de 2018 a 2020 na Biblioteca Virtual em Saúde, PubMed, Science Direct, Scopus e Web of Science. Foram selecionados estudos originais, publicados entre 2007 e 2019, que comparassem o uso dessas tecnologias com o método tradicional da cateterização intravenosa periférica em crianças de 0 - 18 anos. Resultados: de 52 estudos potenciais, 25 compuseram a amostra final, 10 relacionados ao uso da ultrassonografia vascular e 11 da luz infravermelha e 4 da iluminação transdérmica. Conclusão e implicações para a prática: o ultrassom parece ser a tecnologia mais eficaz para promover a obtenção bem sucedida da cateterização intravenosa periférica, porém há necessidade de realização de mais estudos que determinem melhor a eficácia das tecnologias estudadas na obtenção e manutenção da cateterização intravenosa periférica. Poderá propiciar práticas clínicas baseadas em evidências recentes, melhorando a qualidade da assistência à criança hospitalizada e sua família, através da promoção da segurança do paciente.

Palavras-chave: Ultrassonografia; Transiluminação; Criança; Cateterismo Periférico.

\section{ABSTRACT}

Objective: to verify the influence of vascular ultrasound, infrared light emission, and transdermal lighting on the success of peripheral intravenous catheterization, number of attempts, time to perform the procedure, catheter stay in situ and occurrence of complications in children, when compared to the traditional method. Method: this is an integrative literature review carried out from 2018 to 2020 at the Virtual Health Library, PubMed, Science Direct, Scopus, and Web of Science. Original studies, published between 2007 and 2019, comparing the use of these technologies with the traditional method of peripheral intravenous catheterization in children aged 0 - 18 years, were selected. Results: of 52 potential studies, 25 made up the final sample. Ten were related to vascular ultrasound use, 11 to infrared light and 4 to transdermal lighting. Conclusion and implications for practice: ultrasound seems to be the most effective technology to promote the successful achievement of peripheral intravenous catheterization. However, there is a need for further studies to better determine the effectiveness of the technologies studied in obtaining and maintaining peripheral intravenous catheterization. It may provide clinical practices based on recent evidence improving the quality of care for hospitalized children and their families, by promoting patient safety.

Keywords: Ultrasonography; Transillumination; Child; Peripheral Catheterization.

\section{RESUMEN}

Objetivo: verificar la influencia de la ecografía vascular, la emisión de luz infrarroja y la iluminación transdérmica en el éxito del cateterismo intravenoso periférico, el número de intentos, el tiempo para realizar el procedimiento, la permanencia del catéter in situ y la aparición de complicaciones en los niños, en comparación con el método tradicional. Método: esta es una revisión de literatura integradora llevada a cabo desde 2018 hasta 2020 en la Biblioteca Virtual de Salud, PubMed, Science Direct, Scopus y Web of Science. Se seleccionaron estudios originales publicados entre 2007 y 2019 que compararon el uso de estas tecnologías con el método tradicional de cateterización intravenosa periférica en niños de 0 a 18 años. Resultados: De 52 estudios potenciales, 25 constituyeron la muestra final, 10 relacionados con el uso de ultrasonido vascular y 11 con luz infrarroja y 4 con iluminación transdérmica. Conclusión e implicaciones para la práctica: el ultrasonido parece ser la tecnología más efectiva para promover el logro exitoso del cateterismo intravenoso periférico, sin embargo, se necesitan más estudios para determinar mejor la efectividad de las tecnologías estudiadas para obtener y mantener el cateterismo intravenoso periférico. Puedese proporcionar prácticas clínicas basadas en evidencia reciente, mejorando la calidad de la atención para niños hospitalizados y sus familias, promoviendo la seguridad del paciente.

Palabras clave: Ultrasonografía; Transiluminación; Niño; Cateterismo Periférico. 


\section{INTRODUÇÃO}

Independente de sua condição clínica ou cirúrgica, a criança hospitalizada normalmente necessitará da Terapia Intravenosa (TIV) para a sua recuperação, objetivando a infusão de soluções, medicamentos, nutrientes, sangue e seus derivados, por meio da inserção de cateteres venosos por via periférica ou central. NaTIV, devido à facilidade, baixo custo e tempo para a execução da técnica, o procedimento mais comumente realizado em unidades pediátricas é a Cateterização Intravenosa Periférica (CIP). ${ }^{1}$

Entretanto, muitos pacientes não têm sítios venosos periféricos facilmente localizados, o que pode tornar a CIP difícil ou mesmo impossível pelo método tradicionalmente utilizado nas unidades hospitalares, realizado por meio da inspeção visual e palpação de pontos de referência anatômica, ${ }^{2}$ expondo a criança hospitalizada a diversas tentativas mal sucedidas, o que pode aumentar o risco de complicações, dor, estresse e sofrimento.

Análise secundária de um ensaio clinico randomizado realizado com 169 pacientes cirúrgicos verificou que os cateteres que obtiveram sucesso na punção somente depois da terceira tentativa aumentaram o risco de infiltração em 6 vezes, quando comparados aos que obtiveram em apenas uma tentativa $(p=0,0026) .^{3}$

Por isso, para promover uma prática clínica segura durante a execução da TIV, os estabelecimentos de saúde e seus trabalhadores, em especial os enfermeiros, necessitarão inová-la através da utilização de tecnologias que contribuam para melhorar os resultados assistenciais, mais especificamente, o sucesso da CIP na primeira tentativa e promoção do maior tempo de permanência ${ }^{4}$ in situ do cateter intravenoso.

A ultrassonografia vascular, a emissão de luz próxima a infravermelha e a transiluminação são algumas das tecnologias utilizadas para essa finalidade. ${ }^{5} \mathrm{~A}$ ultrassonografia consiste na visualização de estruturas internas através da emissão de ondas sonoras, que são vibrações mecânicas que induzem refrações e compressões alternadas de qualquer meio físico que atravessam. ${ }^{6}$ Por sua vez, tecnologias que utilizam a luz infravermelha baseiam-se na emissão de um feixe de luz infravermelho direto no local da punção ${ }^{5}$ e a transiluminação consiste na emissão de luz embaixo ou ao redor do sítio de punção para a visualização da rede venosa. ${ }^{7}$

Recente revisão sistemática ${ }^{8}$ publicada em 2017 incluiu 15 ensaios clínicos, randômicos e controlados com crianças e adolescentes, conduzidos entre 1998 e 2014, totalizando 4.665 participantes. Essa revisão objetivou identificar as intervenções associadas ao sucesso da CIP na primeira tentativa em crianças hospitalizadas em unidades de emergência. Os estudos revisados foram realizados na Austrália, Canadá, EUA, França, Holanda e Nova Zelândia em unidades de emergência e centro cirúrgico.

Essa revisão sistemática ${ }^{8}$ comparou o uso de ultrassonografia, luz infravermelha ou transiluminação com o método clínico tradicional e concluiu não haver evidências suficientes para sustentar o uso destas tecnologias na prática clínica durante a inserção de CIP em crianças, objetivando reduzir a dor infantil e o sucesso da primeira tentativa. A revisão supramencionada seguiu os princípios da Biblioteca Crochrane e selecinou estudos publicados entre 1989 e 2014.

Entretanto, é necessário buscar novas evidências científicas publicadas após a revisão sistemática anteriormente citada e que indiquem qual a melhor estratégia para a visualização da rede venosa da criança, estando ela em excelentes condições ou já classificada como de difícil acesso para, então, inseri-las nos cenários de cuidados clínicos e oferecer cuidados mais seguros.

Portanto, acredita-se que o uso de tecnologias possa facilitar a inserção de cateteres intravenosos periféricos, garantir a segurança e o permitir o maior tempo de permanência do dispositivo, sendo uma das soluções para amenizar o sofrimento da criança submetida à CIP e propiciar uma intervenção mais rápida, quando necessária.

Desta forma, o presente estudo teve como objetivo verificar a influência da ultrassonografia vascular, emissão de luz infravermelha e iluminação transdérmica no sucesso da CIP, número de tentativas, tempo para a realização do procedimento, permanência do cateter in situ e ocorrência de complicações em crianças, quando comparadas ao método tradicional.

\section{MÉTODO}

Revisão integrativa da literatura, desenvolvida a partir da estratégia PICO, na qual "P" refere-se à população do estudo (crianças hospitalizadas com necessidade de CIP); “l”, à intervenção estudada (uso da ultrassonografia vascular, emissão luz infravermelha e transiluminação para a CIP); "C", à comparação com outra intervenção (método tradicionalmente utilizado para a CIP); "O”, ao desfecho de interesse (sucesso da CIP, número de tentativas, tempo para a realização do procedimento, permanência do cateter in situ e ocorrência de complicações em crianças, quando comparadas ao método tradicional). ${ }^{9}$

As seguintes etapas foram utilizadas para a construção desta revisão: identificação do tema e seleção da questão de pesquisa, estabelecimento dos critérios de elegibilidade, amostragem da literatura, coleta de dados, análise crítica dos estudos, interpretação dos resultados e apresentação da revisão. ${ }^{10}$

Esta revisão foi baseada na seguinte questão norteadora: qual a influência da ultrassonografia vascular, da emissão de luz infravermelha e transiluminação no sucesso da CIP, número de tentativas, tempo deste procedimento, permanência do cateter in situ e ocorrência de complicações em crianças hospitalizadas, quando comparadas ao método tradicional?

Uma busca eletrônica abrangente foi realizada na Biblioteca Virtual em Saúde, National Library of Medicine (PubMed), Science Direct, Scopus, Web of Science e CINAHL, no período de outubro de 2018 a janeiro de 2020, a partir do cruzamento dos descritores controlados "Criança, Cateterismo Periférico, Efeitos adversos, Ultrassonografia e Transiluminação" presentes no DeCS (Descritores em Ciências da Saúde) e seus correspondentes em inglês e espanhol no Medical Subject Headings (MESH). A lógica booleana foi utilizada com os termos de pesquisa, incluindo: Ultrasonography and child and Catheterization, Peripheral or Peripheral Catheterizations; Transillumination and child and Catheterization, Peripheral or Peripheral Catheterizations; 
Transillumination or Ultrasonography and child and Catheterization, Peripheral or Peripheral Catheterizations and adverse effects.

Os critérios de elegibilidade foram: (1) ser publicado na língua portuguesa, inglesa ou espanhola; (2) ser artigo original; (3) estar disponível na íntegra; (4) ter sido publicado entre os anos de 2007 a 2019; (5) tratar de cateter venoso periférico realizado em crianças na faixa etária de 0- 18 anos; (6) comparar o uso da ultrassonografia vascular, da emissão de luz infravermelha ou transiluminação com o método tradicional de CIP.

Dois avaliadores independentes participaram da coleta, leitura e seleção dos artigos. Inicialmente, foram avaliados os títulos e resumos de todos os artigos encontrado e excluídos aqueles que não correspondiam aos objetivos da presente revisão. Para cada estudo potencial, foi examinado o artigo completo e, por fim, foram avaliados os estudos que se encaixavam nos critérios de inclusão.

Foram extraídas as seguintes informações dos artigos selecionados: (1) título, (2) autores, (3) ano da publicação, (4) país de origem do estudo, (5) objetivo, (6) delineamento do estudo, (7) local de realização do estudo, (8) número de participantes, (9) intervenção utilizada, (10) principais resultados e (11) limitações e conclusão. Essas informações foram transportadas para uma planilha e os seus resultados principais foram analisados e comparados pelas suas convergências e divergências.

\section{RESULTADOS}

Foram encontrados 52 artigos (38 na PubMed e 14 na Science Direct), não sendo encontrados artigos sobre a temática nas demais bases analisadas. Durante a leitura do resumo, foram eliminados 16 por analisarem a população adulta/idosa, 7 , por tratarem de cateter central de inserção periférica, 2, por analisarem apenas competência dos profissionais e 2, por incluírem na amostra a faixa etária acima de 18 anos.

Ao final, foram analisados, 25 artigos, 10 relacionados ao uso da ultrassonografia vascular, ,,11-19 11 relacionados ao uso de dispositivos com emissão de luz infravermelha (3 sobre o AccuVein ${ }^{\circledast},{ }^{20-22} 7$ sobre o VeinViewer ${ }^{87,23-28}$ e 1 sobre VascuLuminator $\left.{ }^{\circledR}\right)^{29}$ e 4 relacionados à transiluminação (LED-powered, ${ }^{30}$ Veinlite ${ }^{\circledR 31,32}$ e luz de fibra ótica). ${ }^{33} \mathrm{Um}$ estudo comparou ao mesmo tempo um dispositivo de emissão de luz infravermelha (VeinViewer ${ }^{\circledR}$ ) e um de transiluminação (WeeSight $\left.{ }^{\circledR}\right){ }^{28} \mathrm{~A}$ caracterização dos estudos está descrita no Quadro 1.

Quadro 1. Caracterização dos artigos selecionados. Feira de Santana, BA, 2020.

\begin{tabular}{|c|c|c|c|}
\hline \multicolumn{2}{|c|}{ Tipo de Estudo/ Tecnologia utilizada } & População/ Amostra/Idade & Autores/Ano de Publicação \\
\hline \multirow{10}{*}{ 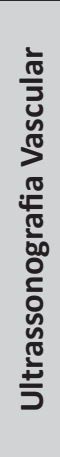 } & Avelar et al. ${ }^{15}$ & ECR/Ultrassom & 335 crianças ( 0 a 18 anos) \\
\hline & Benkhadra et al. ${ }^{11}$ & ECR/Ultrassom & 40 crianças ( $<3$ anos) \\
\hline & Bair et al. ${ }^{12}$ & ECR/Ultrassom & 44 crianças ( $<7$ anos) \\
\hline & Curtis et al. ${ }^{13}$ & ECR/Ultrassom & 428 crianças ( $\leq 16$ anos) \\
\hline & Oakley \& Wong ${ }^{14}$ & Estudo Prospectivo Observacional/Ultrassom & 84 crianças (< 18 anos) \\
\hline & Avelar et al..$^{15}$ & ECR/Ultrassom & 335 crianças ( 12 dias a 17 anos) \\
\hline & Takeshita et al. ${ }^{16}$ & ECR/Ultrassom & 96 crianças ( 1 a 2 anos) \\
\hline & Doniger et al. ${ }^{17}$ & ECR/Ultrassom & 50 crianças (< 10 anos) \\
\hline & Vinograd et al. ${ }^{18}$ & ECR/Ultrassom & 167 crianças ( 0 a 18 anos) \\
\hline & Gopalasingam et al..$^{19}$ & ECR/Ultrassom & 50 crianças ( $>6$ meses e $<4$ anos) \\
\hline \multirow{11}{*}{ 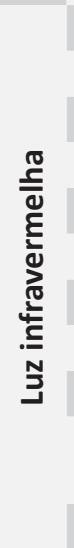 } & Rothbart et al. ${ }^{20}$ & ECR/AccuVein ${ }^{\circledR}$ & 238 crianças ( 0 a 17 anos) \\
\hline & Kaddoum et al. ${ }^{21}$ & ECR/AccuVein ${ }^{\circledR}$ & 146 crianças ( 0 a 17 anos) \\
\hline & Demir et al. ${ }^{22}$ & ECR/AccuVein ${ }^{\circledR}$ & 129 crianças ( 3 a 18 anos_ \\
\hline & Kim et al. ${ }^{7}$ & $\mathrm{ECR} /$ VeinViewer $^{\circledR}$ & 111 crianças ( 1 mês a 16 anos) \\
\hline & Hess $^{23}$ & Estudo prospectivo não randomizado/VeinViewer & 241 crianças (11 dias a 17 anos) \\
\hline & Chapman et al..$^{24}$ & ECR/VeinViewer ${ }^{\circledR}$ & 336 crianças ( 0 a 17 anos) \\
\hline & Szmuk et al. ${ }^{25}$ & $\mathrm{ECR} /$ VeinViewer $^{\circledR}$ & 299 crianças de ( 0 a 18 anos) \\
\hline & Sun et al. ${ }^{26}$ & ECR/VeinViewer ${ }^{\circledR}$ & 60 crianças ( 3 meses a 17 anos) \\
\hline & Perry et al. ${ }^{27}$ & ECR/VeinViewer ${ }^{\circledR}$ & 123 crianças ( 0 a 20 anos) \\
\hline & Peterson et al. ${ }^{28}$ & $\begin{array}{c}\text { Estudo prospectivo não randomizado/WeeSight }{ }^{\circledR} \\
\text { e VeinViewer }\end{array}$ & 546 crianças ( 0 a 3 anos) \\
\hline & Van der Woude et al. ${ }^{29}$ & ECR/VascuLuminator ${ }^{\circledR}$ & 88 crianças ( 0 a 15 anos) \\
\hline \multirow{4}{*}{ 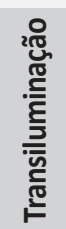 } & Hosokaw et al..$^{30}$ & ECR/(LED)-powered & 136 crianças ( 0 a 3,7 anos) \\
\hline & Katsogridakis et al..$^{31}$ & ECR/Veinlite ${ }^{\circledR}$ & 240 crianças (5 dias a 15 anos) \\
\hline & Gümüş et al. ${ }^{32}$ & ECR/Veinlite ${ }^{\circledR}$ & 112 crianças ( 1 a 10 anos) \\
\hline & Yamazaki et al..$^{33}$ & $\begin{array}{l}\text { ECR/Luz Transmitida } \\
\text { Luz de fibra ótica }\end{array}$ & 100 crianças ( 2 a 5 anos) \\
\hline
\end{tabular}

Fonte: elaborado pelos autores. 
A maioria dos estudos tratava-se de Ensaio Clínico Randomizado (ECR) (22 estudos), porém apenas um dos estudos foi observacional, ${ }^{14} \mathrm{e}$ dois foram ensaios clínicos, mas não randomizados. ${ }^{23,28}$ Os estudos utilizados foram predominantemente internacionais, realizados nos seguintes países: Estados Unidos, ${ }^{11,13,16,18,24,25,28,31}$ Japão, ${ }^{15,29,31}$ Alemanha, ${ }^{15,24}$ Turquia, ${ }^{22,32}$ França, ${ }^{9}$ Canadá, ${ }^{11}$ Austrália ${ }^{21}$ e Tailândia,, 25 sendo encontrados apenas dois estudos nacionais, ambos relacionados ao uso do ultrassom. ${ }^{5,15}$

Foram encontrados poucos estudos sobre o uso das tecnologias, principalmente a ultrassonografia vascular e o AccuVein $^{\circledR}$, o que reforça a necessidade de realização de mais ensaios clínicos randomizados sobre essas tecnologias. Nenhum estudo abordava o uso do venoscópio como tecnologia para a transiluminação, o que pode ser devido à escassez de investigações nacionais sobre o uso de tecnologias para visualização da rede venosa periférica como auxilio na realização da CIP.

Quanto à unidade, a maioria dos estudos foi realizada no departamento de emergência pediátrica, ${ }^{12-18,21,24,30,32}$ seguido pelas unidades cirúrgicas: centro cirúrgico e internamento, ${ }^{5,11,15,16,19,20,28,29}$ o que pode ser justificado pelo maior volume de CIP a necessidade de maior velocidade na sua realização e também a maior destreza exigida por conta das condições clinicas do paciente, que tornam o procedimento mais complexo. ${ }^{34}$

Apenas dez estudos delimitaram os limites inferiores exatos de idade dos seus participantes. . $^{715,16,19,22,23,26,31-33}$ Dentre eles, três incluíram neonatos, lactentes, pré-escolares, escolares e adolescentes no mesmo estudo. 15,23,31 Dois incluíram lactentes, pré-escolares, escolares e adolescentes, ${ }^{7,26}$ dois incluíram pré-escolares e escolares, ${ }^{19,33}$ um incluiu lactentes, pré-escolares e escolares ${ }^{32}$ e outro incluiu pré-escolares, escolares e adolescentes. ${ }^{22}$ Entretanto, apenas um deles separou as faixas etárias no momento da análise de seus desfechos. ${ }^{15}$ Os 15 demais estudos delimitaram apenas o limite superior de idade de seus participantes e não analisaram os grupos etários separadamente em seus resultados.

A variação entre as faixas etárias dos participantes interfere na fidedignidade dos resultados apresentados, já que a idade da criança influencia no sucesso da CIP, pois devido à variação anatômica, as crianças mais velhas têm menos risco para o insucesso na CIP. ${ }^{34}$

As variáveis mais estudadas foram a taxa de sucesso na primeira tentativa, ${ }^{5,7,11-14,18-27,29-32}$ seguida pelo tempo do procedimento, $7,11,13,14,16-22,24,26,29,30,32$ a taxa de sucesso global5, ${ }^{511,12,14,16-18,28,31,33}$ e o número de tentativas. ${ }^{11,13,17-24,26,32}$ Apenas dois estudos analisaram a ocorrência de complicações (infiltração e flebite) ${ }^{15,18}$ e dois analisaram o tempo de permanência do cateter, ${ }^{5,18}$ todos relacionados ao uso do ultrassom.

Observa-se que nem todos os estudos analisaram todas as variáveis, demonstrando lacunas que ainda existem na literatura, principalmente sobre a ocorrência de complicações e o tempo de permanência do cateter, visto que estas foram analisadas em apenas dois estudos. A síntese dos artigos está descrita no Quadro 2.

Quadro 2. Síntese dos artigos selecionados. Feira de Santana, BA, 2020

\begin{tabular}{|c|c|c|}
\hline Autores & Objetivos & Resultados \\
\hline Avelar et al. ${ }^{15}$ & $\begin{array}{l}\text { Verificar se a ultrassonografia vascular aumenta } \\
\text { a assertividade na utilização do CIP e o tempo } \\
\text { de permanência do cateter quando comparado } \\
\text { ao método tradicional de punção. }\end{array}$ & $\begin{array}{l}\text { Não foram observadas diferenças significativas } \\
\text { na taxa de sucesso global }(p=0,970) \text {, na taxa } \\
\text { de sucesso na primeira tentativa }(p=0,232) \text {, } \\
\text { nem no tempo de permanência do cateter } \\
(p=0,121) \text {. }\end{array}$ \\
\hline Benkhadra et al. ${ }^{11}$ & $\begin{array}{l}\text { Comparar o uso do ultrassom vascular com } \\
\text { a técnica padrão em crianças }<3 \text { anos que } \\
\text { necessitam de anestesia geral. }\end{array}$ & $\begin{array}{l}\text { O ultrassom reduziu o tempo médio da } \\
\text { CIP ( } 63,5 \text { segundos versus } 420,5 \text { segundos, } \\
p<0,001) \text {; o número médio de punções } \\
\text { (1 versus } 2,5, p=0,004) \text {; e aumentou a taxa de } \\
\text { sucesso na primeira tentativa ( } 85 \% \text { versus } 35 \% \text {, } \\
p=0,0012) \text {, quando comparado ao método } \\
\text { tradicional. A taxa de sucesso global não diferiu } \\
\text { significativamente entre os grupos ( } 90 \% \text { versus } \\
85 \% \text {, ultrassom versus técnica padrão, } p=0,63 \text { ) }\end{array}$ \\
\hline Bair et al. ${ }^{12}$ & $\begin{array}{l}\text { Determinar se o uso de uma técnica de } \\
\text { ultrassom estática poderia: a) permitir aos } \\
\text { clínicos visualizar veias periféricas; b) melhorar } \\
\text { as taxas de sucesso da CIP. }\end{array}$ & $\begin{array}{l}\text { Os grupos que utilizaram o ultrassom } \\
\text { obtiveram a mesma taxa de sucesso quando } \\
\text { comparados ao método tradicional ( } 57 \% \text { ). } \\
\text { Porém, o sucesso da CIP na primeira tentativa } \\
\text { foi maior no grupo de ultrassom, quando } \\
\text { comparados ao método tradicional (35\%, } \\
\text { versus } 29 \% \text { ). }\end{array}$ \\
\hline
\end{tabular}

Fonte: elaborado pelos autores. 
Quadro 2. Continuação...

\begin{tabular}{|c|c|c|}
\hline Autores & Objetivos & Resultados \\
\hline Curtis et al. ${ }^{13}$ & $\begin{array}{l}\text { Determinar se o uso de ultrassom ou } \\
\text { infravermelho para orientar a CIP seria mais } \\
\text { eficaz do que o método padrão na obtenção de } \\
\text { um cateter bem sucedido na primeira tentativa. }\end{array}$ & $\begin{array}{l}\text { Não foram observadas diferenças significativas } \\
\text { na taxa de sucesso na primeira tentativa - } \\
\text { população geral: }(p=0,3) \text { menores de } 3 \text { anos: } \\
(p=0,2) \text {; nem no tempo de procedimento } \\
(p<0,5) \text {, assim como o número de tentativas } \\
\text { tanto para a amostra total (Ultrassom: } 1 \text { versus } \\
\text { método tradicional: } 1) \text { quanto em menores } \\
\text { de } 3 \text { anos (Ultrassom: } 1,5 \text { versus método } \\
\text { tradicional: } 1) \text {. }\end{array}$ \\
\hline Oakley \& Wong ${ }^{14}$ & $\begin{array}{l}\text { Avaliar a implementação e a utilidade do } \\
\text { ultrassom vascular para auxiliar a CIP em um } \\
\text { departamento de emergência pediátrica. }\end{array}$ & $\begin{array}{l}\text { O ultrassom aumentou a taxa de sucesso na } \\
\text { amostra geral }(42 \% \text { versus } 38 \%, p=0,08 \text { ) e } \\
\text { em pacientes com acesso difícil (sucesso } 35 \% \\
\text { versus } 18 \%, p=0,003) \text {, como também a taxa } \\
\text { de sucesso na tentativa ( } 64 \% \text { versus } 49 \% \text {, } \\
p=0,08) \text {. As tentativas com o uso do ultrassom } \\
\text { vascular demoraram mais do que as tentativas } \\
\text { com o método tradicional ( } 4 \text { minutos versus } \\
2 \text { minutos e } 15 \text { segundos, } p<0,001 \text { ). }\end{array}$ \\
\hline Avelar et al. ${ }^{15}$ & $\begin{array}{l}\text { Analisar se o uso do ultrassom vascular durante } \\
\text { a inserção rotineira de cateteres periféricos } \\
\text { aumenta a taxa de sucesso e reduz a incidência } \\
\text { de infiltração e flebite, em comparação com o } \\
\text { método de referência padrão para inserção do } \\
\text { cateter. }\end{array}$ & $\begin{array}{l}\text { Não foram observadas diferenças significativas } \\
\text { na taxa de sucesso em nenhum dos subgrupos } \\
\text { analisados: }>\mathbf{2} \text { anos }(p=0,148) ; \mathbf{2 - 7} \text { anos } \\
(p=0,848) ; 7-12 \text { anos }(p=0,538) ; 12-18 \text { anos: } \\
(p=0,055) \text {. Já com relação à ocorrência } \\
\text { de complicações, a infiltração aumentou } \\
\text { significativamente com o uso do ultrassom } \\
\text { vascular }(73,9 \% \text { versus } 51,1 \% ; p=0,026) .\end{array}$ \\
\hline Takeshita et al. ${ }^{16}$ & $\begin{array}{l}\text { Examinar os fatores que afetam a CIP de veias } \\
\text { invisíveis e impalpáveis em crianças e avaliar } \\
\text { o melhor sítio para cateterismo guiado por } \\
\text { ultrassom. }\end{array}$ & $\begin{array}{l}\text { O uso do ultrassom vascular aumentou a taxa } \\
\text { de sucesso da CIP ( } 68 \% \text { versus } 39 \% ;(p=0,002) \text { e } \\
\text { reduziu o tempo de procedimento }\left(54^{\prime \prime} \text { versus }\right. \\
\left.180^{\prime \prime} ; p=0,003\right)\end{array}$ \\
\hline Doniger et al. ${ }^{17}$ & $\begin{array}{l}\text { Avaliar se o uso do ultrassom melhora a taxa } \\
\text { de sucesso, reduz o número de tentativas, } \\
\text { o número de redirecionamentos de agulhas } \\
\text { e o tempo total para a CIP em pacientes } \\
\text { pediátricos com acesso difícil em uma } \\
\text { emergência pediátrica. }\end{array}$ & $\begin{array}{l}\text { As taxas globais de sucesso para o grupo } \\
\text { ultrassom foram } 80 \% \text {, e para o grupo controle, } \\
64 \%(p=0,208) .0 \text { ultrassom reduziu o tempo } \\
\text { total }(6,3 \text { versus } 14,4 \text { minutos, } p=0.001 \text { ) e o } \\
\text { número de tentativas (mediana, } 1 \text { contra } 3 ; \\
p=0.004) \text {, quando comparado ao método } \\
\text { tradicional. }\end{array}$ \\
\hline Vinograd et al. ${ }^{18}$ & $\begin{array}{l}\text { Determinar se a ultrassonografia melhora as } \\
\text { taxas de sucesso na primeira tentativa de CIP, } \\
\text { em comparação com métodos tradicionais de } \\
\text { palpação para crianças com acesso difícil. }\end{array}$ & $\begin{array}{l}\text { O ultrassom aumentou a taxa de sucesso } \\
\text { da primeira tentativa ( } 85,4 \% \text { versus } 45,8 \%) \text {, } \\
\text { reduziu o número médio de tentativas } \\
\text { ( } 2 \text { versus } 1 \text { ) e o tempo de realização } \\
\text { do procedimento ( } 28 \text { minutos versus } \\
14 \text { minutos), aumentando a permanência do } \\
\text { cateter ( } 4,5 \text { dias versus } 2,6 \text { dias). Não foram } \\
\text { encontradas diferenças significativas na } \\
\text { ocorrência de complicações ( } 48 \% \text { no grupo } \\
\text { controle versus } 40 \% \text { no grupo ultrassom) } \\
\text { ( } p>0,05) \text {. }\end{array}$ \\
\hline
\end{tabular}

Fonte: elaborado pelos autores. 
Quadro 2. Continuação...

\begin{tabular}{l}
\hline \multicolumn{1}{c}{ Autores } \\
\hline Objetivos \\
Gopalasingam et al. ${ }^{19} \quad \begin{array}{l}\text { Comparar o uso do ultrassom com a técnica } \\
\text { padrão de palpação para CIP em crianças } \\
\text { anestesiadas submetidas a procedimentos } \\
\text { eletivos de baixo risco. }\end{array}$ \\
$\begin{array}{l}\text { Avaliar se o uso do Accuvein }{ }^{\circledR} \text { (AV300) facilita a } \\
\text { CIP em crianças }\end{array}$
\end{tabular}

A taxa de sucesso da primeira tentativa e a taxa de sucesso global foram maiores com o uso do ultrassom ( $84 \%$ versus $60 \% ; p=0,029$ e $100 \%$ versus $84 \%$; $p=0,008$ ), respectivamente. O número de tentativas não obteve diferença significante $(p=0,05)$ e o tempo de realização do procedimento foi ligeiramente maior no grupo ultrassom (192 segundos versus 102 segundos), porém sem significância estatística $(p=0,073)$.

O Accuvein ${ }^{\circledR}$ reduziu o tempo médio da CIP bem sucedida, quando comparado ao método tradicional (1 minutos versus 2 minutos, $p$ $<0,01)$, porém aumentou o número médio de tentativas ( 2 versus $1, p<0,01$ ) e reduziu a taxa de sucesso na primeira tentativa $(0,45 \%$ versus $0,73 \%, p<0,01$ ).

A taxa de sucesso na primeira tentativa não obteve aumento com resultados significantes na amostra geral nem no subgrupo de menores de 2 anos ( $p=0,85$ e 0,62$)$, assim como o número de tentativas $(p=0,86)$ e o tempo de procedimento $(p=0,047)$.

Avaliar a eficácia do Accuvein ${ }^{\circledR}$ (AV300) para

Kaddoum et al. ${ }^{21}$ tentativa da CIP de pacientes pediátricos anestesiados.

Determinar o efeito do uso de um dispositivo de visualização de veias no sucesso do procedimento com base no número de tentativas por paciente, duração do procedimento e a taxa de sucesso na primeira tentativa.

Comparar a taxa de sucesso na primeira

Kim et al. ${ }^{7}$ tentativa da CIP entre o método tradicional e o uso do VeinViewer ${ }^{\circledR}$.

Avaliar a eficácia de um dispositivo de visualização de veias sobre o sucesso das CIP Hess $^{23}$ realizadas por enfermeiros em uma unidade cirúrgica pediátrica

Avaliar se o uso do VeinViewer ${ }^{\circledR}$ reduz o tempo Chapman et al. ${ }^{24}$ e o número de tentativas da CIP em crianças no departamento de emergência pediátrica.
O tempo de procedimento foi menor com o uso do Accuvein ${ }^{\circledR}$ do que com o método tradicional $(37,24$ versus 172,6 segundos; $p=0,001)$, com menos tentativas ( 1,08 versus, 2,23 $p<0,01)$. O uso do Accuvein ${ }^{\circledR}$ também aumentou a taxa de sucesso na primeira tentativa $(91,7 \%$ versus 47,4\%; $p=0,001)$.

O uso do VeinViewer ${ }^{\circledR}$ obteve maior taxa de sucesso na primeira tentativa ( $72 \%$ versus $57 \%$ ), porém sem significância estatística $(p=0,526)$. Ao fazer a regressão logística considerando os pacientes com acessos venosos difíceis, o resultado foi significativo $(p=0,048)$.

O uso do VeinViewer ${ }^{\circledR}$ aumentou a taxa de sucesso na primeira tentativa (80\%), quando comparado ao método tradicional (49\%) $(p<0,01)$, assim como reduziu o número de tentativas de 1,97 para $1,29(p<0,001)$.

A taxa de sucesso na primeira tentativa não obteve resultados significantes nem na amostra geral nem no subgrupo de 0-2 anos ( $p=0,53$ e 0,19 , respectivamente), assim como o número de tentativas $(p=0,3)$ já o tempo de procedimento obteve redução significativa com o uso do VeinViewer ${ }^{\circledR}$ no subgrupo de 0-2 anos (121 minutos versus 167 minutos, $p=0,047$ ). 
Quadro 2. Continuação...

\begin{tabular}{|c|c|c|}
\hline Autores & Objetivos & Resultados \\
\hline Szmuk et al. ${ }^{25}$ & $\begin{array}{l}\text { Avaliar se o VeinViewer }{ }^{\circledR} \text { melhora a taxa de } \\
\text { sucesso na CIP por enfermeiras experientes em } \\
\text { pacientes pediátricos. }\end{array}$ & $\begin{array}{l}\text { O uso do método tradicional de CIP obteve } \\
\text { taxa de sucesso maior }(61 \%) \text { que o uso do } \\
\text { VeinViewer }^{\circledR}(47 \%),(p=0,0003)\end{array}$ \\
\hline Sun et al. ${ }^{26}$ & $\begin{array}{l}\text { Avaliar a eficácia do uso do VeinViewer }{ }^{\circledR} \text { na } \\
\text { visualização da rede venosa em crianças } \\
\text { doentes. }\end{array}$ & $\begin{array}{l}\text { A taxa de sucesso na primeira tentativa foi } \\
\text { a mesma tanto com o uso do VeinViewer } \\
\text { como com o método tradicional }(30 \%) \text {, } \\
\text { porém o número de tentativas e o tempo } \\
\text { de procedimento foram reduzidos com o } \\
\text { uso do VeinViewer }(1 \text { versus } 2, p=0,04) \text { e } \\
(186,16 \text { minutos versus } 427,87 \text { minutos, } \\
p=0.014) \text {, respectivamente. }\end{array}$ \\
\hline Perry et al. ${ }^{27}$ & $\begin{array}{l}\text { Determinar se o uso de dispositivo com luz } \\
\text { infravermelha (VeinViewer }{ }^{\circledR} \text { ) melhora a taxa } \\
\text { de sucesso na primeira tentativa da CIP em um } \\
\text { departamento de emergência pediátrica. }\end{array}$ & $\begin{array}{l}\text { A taxa de sucesso na primeira tentativa foi } \\
\text { maior com o uso do método tradicional }(79 \%) \\
\text { do que como uso do VeinViewer }{ }^{\circledR}(72,1 \%), \\
\text { porém sem significância estatística }(p=0,36) \text {. }\end{array}$ \\
\hline Peterson et al. ${ }^{28}$ & $\begin{array}{l}\text { Examinar o sucesso na visualização de veias } \\
\text { por dois dispositivos auxiliares da CIP por } \\
\text { enfermeiros e médico cirúrgicos pediátricos. }\end{array}$ & $\begin{array}{l}\text { O uso do WeeSight }{ }^{\circledR} \text { e do VeinViewer }{ }^{\circledR} \text { obteve } \\
\text { taxas de sucesso próximas }(35,3 \% \text { e } 32,3 \% \text {, } \\
\text { respectivamente) e inferiores às obtidas com o } \\
\text { uso do método tradicional }(52,2 \%),(p=0,001) \text {. }\end{array}$ \\
\hline Van der Woude et al. ${ }^{29}$ & $\begin{array}{l}\text { Verificar a eficácia de um dispositivo de } \\
\text { infravermelho (VascuLuminator }{ }^{\circledR} \text { ) para facilitar } \\
\text { a CIP em crianças com cor de pele escura. }\end{array}$ & $\begin{array}{l}\text { O uso do VascuLuminator }{ }^{\circledR} \text { aumentou a taxa } \\
\text { de sucesso da primeira tentativa ( } 63 \% \text { versus } \\
51 \% \text { ) e reduziu o tempo de procedimento } \\
\text { ( } 53 \text { segundos versus } 68 \text { segundos), quando } \\
\text { comparado ao grupo controle, porém não } \\
\text { obteve resultados significativos ( } p=0,27 \text { e } 0,50 \text {, } \\
\text { respectivamente). Já no subgrupo com acesso } \\
\text { venoso difícil, o VascuLuminator }{ }^{\circledR} \text { aumentou } \\
\text { significativamente a taxa de sucesso na } \\
\text { primeira tentativa }(p=0,03) \text {. }\end{array}$ \\
\hline Hosokaw et al. ${ }^{30}$ & $\begin{array}{l}\text { Comparar a taxa de sucesso e o tempo } \\
\text { de duração da CIP com e sem o uso da } \\
\text { transiluminação. }\end{array}$ & $\begin{array}{l}\text { A taxa de sucesso com a transiluminação foi } \\
\text { maior }(75 \%) \text {, quando comparado ao método } \\
\text { tradicional }(61 \%) \text {, porém sem significância } \\
\text { estatística }(p=0,10) \text {, já nos menores de } \\
2 \text { anos, a transiluminação obteve significância } \\
\text { estatística }(p=0,03) \text {. Quanto ao tempo de } \\
\text { procedimento, o uso da teransiluminação o } \\
\text { reduziu significativamente }(p=0,01) \text {. }\end{array}$ \\
\hline Katsogridakis et al. ${ }^{31}$ & Avaliar a CIP com o uso do Veinlite ${ }^{\circledR}$. & $\begin{array}{l}\text { A taxa de sucesso em duas tentativas foi maior } \\
\text { com o uso do Veinlite }{ }^{\circledR}(59,5 \% \text { versus } 56,3 \% \text {, } \\
p=0,01) \text {, já o sucesso na primeira tentativa, só } \\
\text { obteve significância estatística após a regressão } \\
\text { logística }(p=0,03) \text {. }\end{array}$ \\
\hline Gümüş et al. ${ }^{32}$ & $\begin{array}{l}\text { Avaliar a eficácia do Veinlite }{ }^{\circledR} \text { PEDI, para } \\
\text { melhorar a visualização das veias no } \\
\text { departamento de emergência pediátrica. }\end{array}$ & $\begin{array}{l}\text { O Veinlite }{ }^{\circledR} \text { aumentou a taxa de sucesso da } \\
\text { primeira tentativa }(92,9 \% \text { versus } 72,2 \% \text {, } \\
p<0,004) \text { e reduziu o número de tentativas } \\
1,07 \text { versus } 1,31 p=0,04) \text { e o tempo total de } \\
\text { tentativas em comparação com grupo controle } \\
(49,98 \text { versus } 59,68 \text { segundos } p=0,01)\end{array}$ \\
\hline
\end{tabular}

Fonte: elaborado pelos autores. 
Quadro 2. Continuação...

\begin{tabular}{cll}
\hline \multicolumn{1}{c}{ Autores } & \multicolumn{1}{c}{ Objetivos } & \multicolumn{1}{c}{ Resultados } \\
\hline & Avaliar as taxas de sucesso a CIP em crianças & O uso da transiluminação com luz de fibra \\
Yamazaki et al. ${ }^{33}$ & com o uso da transiluminação. & $\begin{array}{l}\text { oumentou a taxa de sucesso da CIP } \\
(p=0,0000002) .\end{array}$ \\
\hline
\end{tabular}

Fonte: elaborado pelos autores.

\section{DISCUSSÃO}

\section{Taxa de sucesso global}

A taxa de sucesso global da luz infravermelha foi analisada em um estudo (VeinViewer $\left.{ }^{\circledR}\right)^{28}$ e da transiluminação foi analisada em três estudos (WeeSight ${ }^{\circledR},{ }^{28}$ Veinlite ${ }^{\circledR 31}$ e luz de fibra ótica). ${ }^{33}$ Dentre esses últimos, o que utilizou o Veinlite ${ }^{\circledR}$ aumentou essa taxa em $11,1 \%$, quando comparada ao grupo controle $(p=0,01) \cdot{ }^{31}$ Ao utilizar a luz de fibra ótica, a taxa de sucesso global aumentou em 33\% $(p=0,0000002) .33$

Entretanto, o uso do WeeSight ${ }^{\circledR}$ e o VeinViewer ${ }^{\circledR}$ obteve menores taxas de sucesso global, $35,3 \%$ e $32,3 \%$, respectivamente $(p=0,001)$, em comparação ao método clínico tradicional. ${ }^{28}$

Dentre os estudos com o ultrassom, sete deles aumentaram a taxa de sucesso global alcançando resultados entre 43 e $100 \%$ no grupo intervenção. $5,11,12,14,16,17,19$ Entretanto, destes, apenas dois obtiveram significância estatística ao analisar a amostra total $(p=0,002$ e $p=0,008) \cdot{ }^{16,19}$ Ao analisar separadamente os acessos considerados como difíceis, um estudo obteve significância $(p=0,003) .{ }^{14}$ Os artigos que obtiveram significância estatística com o uso do ultrassom aumentaram a taxa de sucesso global em $16 \%,{ }^{19} 17 \%{ }^{14}$ e $19 \%,{ }^{16}$ quando comparados ao grupo controle.

Portanto, parece que dentre as tecnologias estudadas, os equipamentos que utilizam a transiluminação por luz de fibra ótica aumentam mais a taxa de sucesso global da CIP, em comparação a utilização do método tradicional. Pensa-se que o uso dessas tecnologias, em comparação aos equipamentos que funcionam a base de ultrassonografia, são mais portáteis e de fácil manuseio pelo profissional operador da técnica de cateterização.

A movimentação da criança ou seu estresse durante as tentativas de CIP pode influenciar na formação da imagem nos aparelhos estudados comprometendo a taxa de sucesso global principalmente com o uso da luz infravermelha e do ultrassom, sendo que nenhum dos estudos analisados consideraram essa variável.

\section{Taxa de sucesso na primeira tentativa}

A taxa de sucesso na primeira tentativa foi analisada em nove dos estudos sobre a luz infravermelha (AccuVein ${ }^{\circledR}, 20-22$ VeinViewer $^{\circledR}{ }^{7,23-27}$ e Vasculuminator $\left.{ }^{\circledR}\right) .{ }^{29}$

Nos estudos sobre VeinViewer ${ }^{\circledR}$, os resultados com o uso desta tecnologia ficaram entre 47 a $80 \%$, e os resultados do grupo controle, entre 49 a 79\%. ${ }^{7,23-27}$ Destes, somente dois obtiveram significância estatística, sendo que um deles apresentou aumento de $31 \%$ na taxa de sucesso na primeira tentativa entre os participantes que utilizaram a tecnologia, quando comparado ao método tradicional $(p<0,01) \cdot{ }^{23}$ Outro apresentou redução dessa taxa em $15 \%$ em comparação ao grupo controle $(p=0,0003){ }^{25}$

Com relação ao AccuVein ${ }^{\circledR}$, os resultados demonstraram taxa de sucesso na primeira tentativa de 45 a $91,7 \% .{ }^{20-22}$ Porém, só dois obtiveram significância estatística, um aumentou a taxa em $44,3 \%(p=0,001)^{22}$ e o outro apresentou uma redução em $0,28 \%$ no sucesso na primeira tentativa com o uso da tecnologia em comparação ao uso do método tradicional $(p<0,01) \cdot{ }^{20}$ Já o VascuLuminator $^{\circledR}$, aumentou em $12 \%$ a taxa de sucesso na primeira tentativa, porém sem significância estatística $(p=0,27) \cdot{ }^{29}$

Três estudos sobre a transiluminação utilizaram esta variável ( 1 com o LED-powered ${ }^{30}$ e dois com o Veinlite $\left.{ }^{\circledR}\right) .{ }^{31,32}$ O LED-powered aumentou em $14 \%$ o sucesso na primeira tentativa, quando comparado ao método tradicional, porém não obteve significância estatística $(p=0,1) .{ }^{30} \mathrm{O}$ Veinlite ${ }^{\circledR}$ em um estudo aumentou a taxa de sucesso na primeira tentativa em $9,1 \%(p<0,04),{ }^{32}$ mas em outro só obteve significância estatística após regressão logística $(p=0,03) .{ }^{31}$

Sete estudos sobre o ultrassom ${ }^{5,11-14,18,19}$ utilizaram esta variável, obtendo resultados entre 29 a 85,4\%. ${ }^{5,11-14,19,20}$ Porém, destes, apenas três obtiveram resultados estatisticamente significantes revelando um aumento de $50 \%$ ( $p=0,0012),{ }^{11} 39,6 \%$ $(p<0,05)^{19}$ e $24 \% \quad(p=0,09)^{20}$ na taxa de sucesso na primeira tentativa com o uso dessa tecnologia, quando comparados ao método tradicional.

Muitos estudos apresentaram elevações nas taxas de sucesso na primeira tentativa. No entanto, apenas três que utilizaram o ultrassom vascular, ${ }^{11,19,20}$ dois que utilizaram a luz infravermelha (VenViewer ${ }^{\circledR 23}$ e AccuVein ${ }^{\circledR}$ ), ${ }^{22}$ e dois que utilizaram a transiluminação $\left(\text { Veinlite }{ }^{\circledR}\right)^{31,32}$ obtiveram resultados estatisticamente significativos. Sendo assim, é necessária a realização de mais estudos que incluam esta variável em suas análises.

Dentre as tecnologias que obtiveram significância, as que mais aumentaram a taxa de sucesso na primeira tentativa foram o AccuVein ${ }^{\circledR}$ e o ultrassom.

\section{Número de tentativas}

O número de tentativas foi avaliado em seis estudos sobre a luz infravermelha (VeinViewer ${ }^{\circledR 23,24,26}$ e AccuVein $\left.^{\circledR}\right) .{ }^{20-22}$ Com relação ao uso do VeinViewer ${ }^{\circledR}$, dois estudos calcularam a mediana de tentativas, encontrando o mesmo resultado com o uso da tecnologia (mediana 1), ${ }^{24,26}$ porém diferenciaram-se com relação aos resultados no grupo controle obtendo mediana $2(p=0,04)^{26}$ e mediana $1,{ }^{24}$ sendo que esse último não obteve significância 
estatística $(p=0,5) \cdot{ }^{24}$ Outro estudo sobre o Vein Viewer ${ }^{\circledR}$ calculou a média e obteve 1,29 com o uso da tecnologia e 1,97, com o uso do método clínico tradicional $(p<0,001){ }^{23}$

Com relação ao AccuVein ${ }^{\circledR}$, três estudos analisaram esta variável, que demonstraram um aumento no número de tentativas com o uso da tecnologia obtendo mediana 2 com o uso da tecnologia e 1 com o método tradicional; médias $1,33^{21}$ e 1,08, ${ }^{22}$ com o uso da tecnologia; $1,29^{21}$ e $2,23,{ }^{22}$ com o uso do método tradicional, porém apenas dois obtiveram resultados estatisticamente significantes $(p<0,01) .^{20,22}$

Apenas um estudo sobre transiluminação através do aparelho Veinlite ${ }^{\circledR}$ utilizou essa variável, obtendo uma média de 1,07 tentativas com o uso da tecnologia e 1,31 com o método tradicional $(p=0,04){ }^{32}$

Cinco estudos sobre o uso do ultrassom analisaram esta variável. ${ }^{11,13,15,18,19}$ Três deles obtiveram mediana $1,{ }^{11,17,18}$ já o grupo controle obteve medianas de $2,{ }^{18} 2,5^{11}$ e $3,{ }^{17}$ com significância estatística $(p=0,004)^{11,17}$ e $(p<0,05)^{18}$ em seus resultados, reduzindo pela metade ou mais o número de tentativas quando comparados ao grupo controle. Um estudo analisou separadamente pacientes com 16 anos ou menos, 3 anos ou menos e mais de três anos, obtendo medianas de 1, 1,5 e 1, respectivamente, ${ }^{13}$ porém sem significância estatística.

O ultrassom, dentre as tecnologias, foi a que mais obteve resultados significativos quanto à redução do número de tentativas, portanto, parece que, ao compará-lo com o método clínico tradicional de CIP, pode proporcionar benefícios, como o início imediato da TIV, minimização do estresse da criança e de seus familiares e ajuda a preservar a anatomia vascular dos danos causados por esse procedimento.

\section{Tempo de procedimento}

O tempo de procedimento foi avaliado em sete estudos sobre a luz infravermelha (VeinViewer ${ }^{\circledR}, 7,21,24,26$ AccuVein ${ }^{\circledR 20-22}$ e Vasculuminator $\left.{ }^{\circledR}\right) .{ }^{29}$ O VeinViewer ${ }^{\circledR}$ reduziu o tempo de procedimento, quando comparado ao método tradicional, $7,21,24,26$ porém a significância estatística foi observada em apenas dois estudos: um apresentou redução de 311,05 segundos no tempo do procedimento, em comparação com o grupo controle $(p=0,014)^{26}$ e o outro apresentou redução de 46 segundos no subgrupo formado por crianças menores de dois anos, quando comparado o uso da tecnologia ao método tradicional $(p=0,047) .^{24}$

O AccuVein ${ }^{\circledR}$ também reduziu o tempo do procedimento em todos os estudos, ${ }^{20-22}$ mas observou-se significância estatística em apenas dois estudos, nos quais o uso do AccuVein ${ }^{\circledR}$ reduziu o tempo de procedimento em $60(p<0,01)^{20}$ e 135,6 $(p=0,001)^{22}$ segundos, quando comparados ao método tradicional. Por fim, - Vasculuminator ${ }^{\circledR}$ reduziu o tempo em 15 segundos, quando comparado ao método tradicional, mas sem significância estatística $(p=0,50) \cdot{ }^{29}$

Dois estudos sobre a transiluminação avaliaram essa variável (LED-powered ${ }^{30}$ e Veinlite ${ }^{\circledR 32}$ ). No LED-powered, observou-se uma redução desse tempo em 21 segundos, ao comparar com o método tradicional $(p=0,01) \cdot{ }^{30}$ Já com o uso do Veinlite ${ }^{\circledR}$, esse tempo reduziu em 9,7 segundos $(p=0,01){ }^{32}$

Sete estudos sobre ultrassom analisaram esta variável, obtendo resultados entre 54 a 510 segundos. ${ }^{11,13,14,16-19}$ Destes, cinco encontraram valores estatisticamente significativos constatando que o uso desta tecnologia reduziu o tempo de procedimento em 486 segundos $(p=0,001),{ }^{17} 126$ segundos $(p=0,003),{ }^{16} 105$ segundos $(p<0,001),{ }^{14} 357$ segundos $(p<0,001){ }^{11}$ e 14 minutos $(p<0,05){ }^{18}$

Portanto, parece que dentre as tecnologias, a que mais reduziu o tempo de procedimento, quando comparada ao método tradicional, foi o ultrassom. A redução deste tempo gera intervenções mais rápidas e, desta maneira, melhora a assistência e ameniza o estresse da criança e de seus familiares.

\section{Ocorrência de complicações}

A ocorrência de complicações foi avaliada em apenas dois artigos, ${ }^{15,18}$ sendo que um encontrou maiores taxas de infiltração no grupo com o uso do ultrassom (73,9\%), em comparação ao grupo controle $(51,1 \%)(p=0,026)$, porém as taxas de flebite reduziram com o uso da tecnologia em $11,3 \%$, apesar de não ter alcançado significância estatística $(p=0,09) \cdot{ }^{15} \mathrm{O}$ outro encontrou uma redução de $8 \%$ na ocorrência de complicações com o uso do ultrassom, porém sem significância estatística $(p>0,05) \cdot{ }^{18}$

A redução no número de complicações pode favorecer o aumento no tempo de permanência do cateter, uma vez que não precisará ser retirado antes do término da TIV, o que reduzirá custos extras causados por um novo procedimento, além de reduzir danos à integridade física do paciente contribuindo para a promoção de práticas seguras. A escassez de estudos com esta variável impede a obtenção de resultados mais concretos sobre a redução ou não de complicações, principalmente com relação às tecnologias com base na transiluminação que não foram avaliadas quanto a esta variável em nenhum estudo.

\section{Tempo de permanência do cateter}

Os dois estudos que utilizaram esta variável foram realizados com o ultrassom, ${ }^{5,18}$ sendo que um demonstrou redução no período de permanência do dispositivo com o uso da tecnologia (22,0 horas), quando comparado ao grupo controle (23,1 horas), porém sem significância estatística $(p=0,121) \cdot{ }^{5} \mathrm{O}$ outro encontrou um tempo maior de permanência ( 4,5 dias), em comparação ao grupo controle $\left(2,6\right.$ dias) $(p<0,05) .{ }^{18}$

Portanto, é necessário o desenvolvimento de investigações acerca desta variável, tanto com relação ao ultrassom como às tecnologias com base na transiluminação, a fim de se obter resultados mais concretos para auxílio na prática clínica.

\section{Limitações e vantagens dos estudos}

Os artigos apresentaram diversas limitações que influenciaram os seus resultados finais, sendo as mais comuns o tamanho da amostra, dada como pequena ou insuficiente em oito estudos; ${ }^{12-15,17,20,23,30}$ a ampliação da veia muito além do seu tamanho normal pelo dispositivo, dando uma falsa ideia de profundidade e favorecendo a ocorrência de transfixação, apontada por cinco estudos; ${ }^{7,20,21,25,27}$ e a diferença nas faixas etárias entre as crianças dos grupos controle e intervenção, também em cinco dos estudos analisados nesta revisão. ${ }^{5,14,15,23,28}$ 
Apesar dessas limitações, as tecnologias apresentaram muitas vantagens, quando comparadas ao método tradicional da $\mathrm{CIP}$, pois aumentam o sucesso na primeira tentativa e reduzem o tempo de procedimento e o número de tentativas. Por conseguinte, preservarão a integridade do endotélio vascular e diminuirão os episódios de estresse e sofrimento que esse procedimento causa tanto à criança, quanto aos seus familiares, promovendo a segurança do paciente.

\section{CONCLUSÃO}

A utilização de tecnologias para visualização direta da rede venosa possibilita uma identificação mais rápida e precisa do sítio de inserção, quando comparadas às técnicas padrão que são baseadas na anatomia superficial e estimam a localização do vaso. Dessa forma, reduzem o tempo e o número de tentativas, principalmente, em redes venosas consideradas de difícil cateterização.

Dentre as tecnologias discutidas por este estudo, o ultrassom parece ser a tecnologia mais eficaz para promover a obtenção bem sucedida da CIP, uma vez que alcançou resultados mais satisfatórios quanto ao sucesso na primeira tentativa, tempo de procedimento e número de tentativas em comparação ao método clínico tradicional.

Porém, a incipiente produção e publicação do conheciemento dificultou a obtenção de resultados mais conclusivos para melhor determinar a eficácia das tecnologias estudadas na obtenção e manutenção da CIP. Quanto à literatura nacional, percebeu-se que há poucos estudos com essa temática, ressaltando as tecnologias de transiluminação, já que não foi encontrada nenhuma pesquisa.

Além disso, a maioria dos estudos analisou a realidade da emergência pediátrica, sendo necessária, portanto, a realização de pesquisas em outros contextos de realidade clínica como, por exemplo, as unidades médica, cirúrgica e oncológica, a fim de compreender a execução da CIP em situações diversas de atenção à criança hospitalizada.

Portanto, esta revisão gerou novas inquetações que evidenciam a necessidade de realizar outros ensaios clínicos sobre o uso dessas tecnologias, a fim de reduzir as limitações e preencher as lacunas existentes na literatura acerca da temática, além de propiciar práticas clínicas baseadas em evidências recentes, melhorando a qualidade da assistência à criança hospitalizada e sua família, através da promoção da segurança do paciente.

\section{CONTRIBUIÇÕES DOS AUTORES}

Desenho do estudo de revisão, aquisição, análise de dados e interpretação dos resultados, redação e revisão crítica do manuscrito, aprovação da versão final do artigo, responsabilidade por todos os aspectos do conteúdo e a integridade do artigo publicado: Sarah Almeida Santos e Luciano Marques dos Santos. Análise de dados e interpretação dos resultados, redação e revisão crítica do manuscrito, aprovação da versão final do artigo, responsabilidade por todos os aspectos do conteúdo e a integridade do artigo publicado: Ariane Ferreira Machado Avelar, Bianka Souza Martins Silva e Rosana Castelo Branco de Santana

\section{EDITOR ASSOCIADO}

\author{
Marcelle Miranda da Silva
}

\section{REFERÊNCIAS}

1. Harada M, Pedreira M. Terapia intravenosa e infusões. $1^{a}$ ed. São Paulo: Yendis Editora; 2011.

2. Blaivas $M$, Lyon M. The effect of ultrasound guidance on the perceived difficulty of emergency nurse-obtained peripheral IV access. J Emerg Med 2006;31(4):407-10. http://dx.doi.org/10.1016/j.jemermed.2006.04.014 PMid:17046483.

3. Johann DA, Danski MTR, Vayego SA, Barbosa DA, Lind J. Fatores de risco para complicações no cateter venoso periférico em adultos: análise secundária de ensaio clinico randomizado. Rev Latino-Am Enfermagem. 2016;24(e2833):2-10.

4. Avelar A, Peterlini M, Pedreira M. Assertividade e tempo de permanência de cateteres intravenosos periféricos com inserção guiada por ultrassonografia em crianças e adolescentes. Rev Esc Enferm USP. 2013;47(3):539-46. http://dx.doi.org/10.1590/S0080-623420130000300003. PMid:24601127.

5. Pitts S, Ostroff M. The use of visualization technology for the insertion of peripheral intravenous catheters. JAVA. 2019;24(3):10-4. http://dx.doi. org/10.2309/j.java.2019.003.007.

6. Flato UA, Petisco G, Santos F. Punção venosa guiada por ultra-som em unidade de terapia intensiva. Rev Bras Ter Intensiva. 2009;21(2):190-6. http://dx.doi.org/10.1590/S0103-507X2009000200012. PMid:25303350.

7. Kim MJ, Park JM, Rhee N, Je SM, Hong SH, Lee YM et al. Efficacy of VeinViewer in pediatric peripheral intravenous access: a randomized controlled trial. Europ Jour Ped. 2012;171(7):1121. http://dx.doi. org/10.1007/s00431-012-1713-9.

8. Parker SI, Benzies KM, Hayden KA. A systematic review: effectiveness of pediatric peripheral intravenous catheterization strategies. J Adv Nurs. 2017;73(7):1570-82. http://dx.doi.org/10.1111/jan.13211.PMid:27864995.

9. Nobre CRM, Bernardo WM, Jatene BF. Prática clínica baseada em evidências: parte I - questões clínicas bem construídas. Rev Bras Reomato. 2004;44(6):397-402. http://dx.doi.org/10.1590/S0482 50042004000600002.

10. Botelho LLR, Cunha CCA, Macedo M. The integrative review method in organizational studies. Rev Eletr Gestão Soc, 2011;5(11):121-36. retrotraduzido por: David Harrad. Epidemiol Serv Saude. 2015;24(2):335-42.

11. Benkhadra M, Collignon M, Fournel I, Oeuvrard C, Rollin P, Perrin $M$ et al. Ultrasound guidance allows faster peripheral IV cannulation in children under 3 years of age with difficult venous access: a prospective randomized study. Paediatr Anaesth. 2012;22(5):449-54. http://dx.doi. org/10.1111/j.1460-9592.2012.03830.x. PMid:22409596.

12. Bair AE, Rose JS, Vance CW, Andrada-Brown E, Kuppermann N Ultrasound-assisted peripheral venous access in young children: a randomized controlled trial and pilot feasibility study. West $\mathrm{J}$ Emerg Med. 2008;9(4):219-24. PMid:19561750.

13. Curtis SJ, Craig WR, Logue E, Vandermeer B, Hanson A, Klassen T. Ultrasound or near-infrared vascular imaging to guide periphera intravenous catheterization in children: a pragmatic randomized controlled trial. CMAJ. 2015;187(8):563-70. http://dx.doi.org/10.1503/ cmaj.141012. PMid:25897047.

14. Oakley E, Wong A. Ultrasound-assisted peripheral vascular access in a paediatric ED. Emerg Med Australas. 2010 jan 26;22(2):166-70. PMid:20534052.

15. Avelar AFM, Poterllini MAS, Pedreira MLG. Ultrasonography-guided peripheral intravenous access in children: a randomized controlled trial. J Infus Nurs. 2015;38(5):320-7. http://dx.doi.org/10.1097/ NAN.0000000000000126. PMid:26339938.

16. Takeshita J, Nakayama Y, Nakajima Y, Sessler DI, Ogawa S, Sawa $\mathrm{T}$ et al. Optimal site for ultrasound-guided venous catheterisation in 
paediatric patients: na observational study to investigate predictors for catheterisation success and a randomised controlled study to determine the most successful site. Crit Care. 2015;19(1):15. http:// dx.doi.org/10.1186/s13054-014-0733-4. PMid:25600063.

17. Doniger SJ, Ishimine P, Fox JC, Kanegaye JT. Randomized controlled trial of ultrasound-guided peripheral intravenous catheter placement versus traditional techniques in difficult-access pediatric patients Pediatr Emerg Care. 2009;25(3):154-9. http://dx.doi.org/10.1097/ PEC.0b013e31819a8946. PMid:19262420.

18. Vinograd AM, Chen AE, Woodford AL, Fesnak S, Gaines S, Elci O et al Ultrasonographic guidance to improve first-attempt success in children with predicted difficult intravenous access in the emergency department: a randomized controlled trial. Ann Emerg Med. 2019;74(1):19-27. http:// dx.doi.org/10.1016/j.annemergmed.2019.02.019. PMid:31126618.

19. Gopalasingam N, Obad DS, Kristensen BS, Lundgaard P, Veien M, Gjedsted $\mathrm{J}$ et al. Ultrasound-guidance outperforms the palpation technique for peripheral venous catheterisation in anaesthetised toddlers: a randomised study. Acta Anaesthesiol Scand. 2017;61(6):601-8. http:// dx.doi.org/10.1111/aas.12901. PMid:28485467.

20. Rothbart A, Yu P, Muller-Lobeck L, Spies CD, Wernecke KD, Nachtigall I. Peripheral intravenous cannulation with support of infrared laser vein viewing system in a pre-operation setting in pediatric patients. BMC Res Notes. 2015;8:463. http://dx.doi.org/10.1186/s13104-015-1431-2. PMid:26391665.

21. Kaddoum RN, Anghelescu DL, Parish ME, Wright BB, Trujillo L, Wu $\mathrm{J}$ et al. A randomized controlled trial comparing the AccuVein AV300 device to standard insertion technique for intravenous cannulation of anesthetized children. Paediatr Anaesth. 2012;22(9):884-9. http:// dx.doi.org/10.1111/j.1460-9592.2012.03896.x. PMid:22694242.

22. Demir D, Inal $S$. Does the use of a vein visualization device for peripheral venous catheter placement increase success rate in pediatric patients? Pediatr Emerg Care. 2019;35(7):474-9. http://dx.doi.org/10.1097/ PEC.0000000000001007. PMid:28072667.

23. Hess HÁ. A biomedical device to improve pediatric vascular access success. Pediatr Nurs. 2010;36(5):259-263. PMid:21067078.

24. Chapman LL, Sullivan B, Pacheco AL, Draleau CP, Becker BM. VeinViewer-assisted intravenous catheter placement in a pediatric emergency children. Ped Neo. 2012;2013(54):194-7.

25. Szmuk P, Steiner J, Pop RB, Farrow-Gillespie A, Mascha EJ, Sessler DI. The veinviewer vascular imaging system worsens first-attempt cannulation rate for experienced nurses in infants and children with anticipated difficult intravenous access. Anesth Analg. 2013;116(5):1087-92. http:// dx.doi.org/10.1213/ANE.0b013e31828a739e. PMid:23492965.

26. Sun CY, Lee KC, Lin IH, Wu CL, Huang HP, Lin YY et al. Near-infrared light device can improve intravenous cannulation in critically ill system in a pre-operation setting in pediatric patients. Res Notes. 2015;8(463):2-6.

27. Perry AM, Caviness AC, Hsu DC. Efficacy of a near-infrared light device in pediatric intravenous cannulation: a randomized controlled trial. Pediatr Emerg Care. 2011;27(1):5-10. http://dx.doi.org/10.1097/ PEC.0b013e3182037caf. PMid:21178814.

28. Peterson KA, Phillips AL, Truemper E, Agrawal S. Does the use of an assistive device by nurses impact peripheral intravenous catheter insertion success in children? J Pediatr Nurs. 2012;27(2):134-43. http:// dx.doi.org/10.1016/j.pedn.2010.10.009. PMid:22341192.

29. Van der Woude Olga CP, Cuper NJ, Getrouw C, Kalkman CJ, Graaff JC. The effectiveness of a near-infrared vascular imaging device to support intravenous cannulation in children with dark skin color: a cluster randomized clinical trial. Anesth Analg. 2013;116(6):1266-71. http://dx.doi.org/10.1213/ANE.0b013e31828e5bde. PMid:23649104.

30. Hosokawa K, Kato H, Kishi C, Kato Y, Shime N. Transillumination by light-emitting diode facilitates peripheral venous cannulations in infants and small children. Acta Anaesthesiol Scand. 2010;54(8):957-61. http:// dx.doi.org/10.1111/j.1399-6576.2010.02270.x. PMid:20626357.

31. Katsogridakis YL, Seshadri R, Sullivan C, Waltzman ML. Veinlite transillumination in the pediatric emergency department a therapeutic interventional trial. Pediatr Emerg Care. 2008;24(2):83-8. http://dx.doi. org/10.1097/PEC.0b013e318163db5f. PMid:18277843.

32. Gümüș $M, B a s ̧ b a k k a l ~ Z$. Efficacy of veinlite $P E D I$ in pediatric peripheral intravenous access a randomized controlled trial. Pediatr Emerg Care. 2018. In press. http://dx.doi.org/10.1097/PEC.0000000000001515. PMid:29794951.

33. Yamazaki S, Tomita S, Watanabe M, Kawaai H, Shimamura K. Effects of a transmitted light device for pediatric peripheral venipuncture and intravenous cannulation. Med Devices. 2011;4(1):189-92. http://dx.doi. org/10.2147/MDER.S18497. PMid:22915945.

34. Yen K, Riegert A, Gorelick MH. A clinical prediction rule for the identification of childrenwith difficult intravenous access. Pediatr Emerg Care 2008;24(3):143-7. http://dx.doi.org/10.1097/PEC.0b013e3181666f32. PMid:18347490. 